\title{
Anestesia y ventilación unipulmonar para bullectomía mínimamente invasiva. A propósito de un caso
}

\author{
Case report: Anesthesia and single lung ventilation \\ for minimally invasive bullectomy
}

Guillermo Capote Guerrero', Domingo Ángel Labrada Tapia², Martha Rosa Rosabal Sadín³, Ariadna Gorgoso Vázquez ${ }^{4}$, Hisyobis Cárdenas Suri ${ }^{5}$

\begin{abstract}
Introduction: Chronic obstructive pulmonary disease is an underdiagnosed disease; it is one of the pulmonary diseases with the greatest impact on health worldwide. Objective: To describe the anesthetic conduct carried out in a patient who undergoes surgical intervention to practice bullectomy of the right lung. Clinical case: We present the case of a 42-year-old smoker who was admitted a month ago with bilateral pneumothorax due to emphysematous bullae. He underwent anesthesia and multimodal analgesia to undergo bullectomy, achieving a good recovery and without complications. Conclusions: The use of controlled ventilation strategies in volume control mode regulated by pressure. Combined anesthesia and multimodal analgesia as well as respiratory physiotherapy in patients who will undergo surgery to perform bullectomy is a good anesthetic strategy that guarantees an adequate recovery of the patient.
\end{abstract}

\section{Key words:}

Anesthesia for

bullectomy,

bullae,

pneumothorax

Especialista de segundo grado en Anestesiología y Reanimación. Diplomado en terapia intensiva y emergencias. Profesor auxiliar. Hospital General docente Carlos Manuel de Céspedes.

2 Especialista de segundo grado en Anestesiología y Reanimación. Verticalizado en cuidados intensivos del adulto. Máster en urgencias médicas. Profesor auxiliar. Hospital General docente Carlos Manuel de Céspedes.

3 Especialista de segundo grado en Anestesiología y Reanimación. Máster en urgencias médicas. Profesor asistente. Hospital General docente Carlos Manuel de Céspedes.

$4 \quad$ Especialista de primer grado en Cirugía General. Hospital General docente Carlos Manuel de Céspedes.

5 Especialista de primer grado en MGl y Terapia Intensiva y Emergencias. Instructor. Hospital Anldo Millán Castro. Santa Clara.

Fecha de recepción: 10 de agosto de 2019

Fecha de aceptación: 22 de enero de 2020

\section{ORCID}

http://orcid.org/0000-0003-2841-8348

Correspondencia:

Guillermo Capote Guerrero

gcapote82@gmail.com 


\section{RESUMEN}

Introducción: La enfermedad pulmonar obstructiva crónica es una enfermedad infradiagnosticada, es uno de los padecimientos pulmonares con mayor repercusión en la salud a nivel mundial. Objetivo: Describir la conducta anestésica llevada a cabo en un paciente que se interviene quirúrgicamente para practicarle bullectomía de pulmón derecho. Caso clínico: Se presenta el caso de un paciente de 42 años de edad, fumador que ingresa hace un mes con neumotórax bilateral por bullas enfisematosas, se le realiza anestesia y analgesia multimodal, para realizarle bullectomía, logrando una buena recuperación del mismo y sin complicaciones. Conclusiones: La utilización de estrategias de ventilación controladas en modalidad volumen control regulada por presión. Anestesia combinada y analgesia multimodal, además, de una fisioterapia respiratoria en pacientes que serán operados para realizarles bullectomía es una buena estrategia anestésica que garantiza una adecuada recuperación del enfermo.

\section{Palabras clave:}

Anestesia para

bullectomía,

bullas,

neumotórax

\section{Introducción}

Lex a enfermedad pulmonar obstructiva crónica es una enfermedad infradiagnosticada, con una -elevada morbimortalidad y supone un problema de salud pública de gran magnitud[1]. En Latinoamérica se estima que $13,6 \%$ de las personas mayores de 40 años la padece[2]

El enfisema centrolobulillar es la forma de lesión pulmonar más frecuente asociada al tabaquismo. Afecta a más de la mitad de los fumadores[3].

La bulla es una lesión pulmonar radiotransparente bien definida. La bullectomía es la técnica quirúrgica que se realiza para extirpar las bullas. En la actualidad se realiza mediante una pequeña incisión de toracotomía que se asiste por video cirugía[4].

Constituye el objetivo de este trabajo describir la conducta anestésica llevada a cabo en un paciente que se interviene quirúrgicamente para practicarle bullectomía de pulmón derecho.

\section{Presentación de caso}

Paciente masculino de 42 años, fumador hace 25 años e hipertenso. Hace 5 años se le diagnosticó la presencia de bullas enfisematosas en ambos pulmones. Hace 2 años fue ingresado por la ruptura de una bulla en el pulmón izquierdo para lo cual se le realizó una pleurostomía mínima alta, resolviendo después de algunos días el proceso.

Ahora después de un mes ingresado por presentar nuevamente ruptura de otras bullas, pero en ambos pulmones para lo cual se le realiza pleurostomía por sonda alta en ambos hemitórax sin lograr resolución del problema. Se le realiza pleurodesis y tampoco se logran resultados favorables. El paciente se mantiene acoplado a equipo de Overholt para lograr expandir los pulmones, en el pulmón izquierdo se ha logrado cierto grado de resolución, pero no así con el derecho, por tal motivo se hace necesario corregir quirúrgicamente el problema.

Se anuncia para ser operado por cirugía mínimamente invasiva y practicarle bullectomía derecha.

\section{Interrogatorio}

Presenta falta de aire moderada la cual se intensifica cuando es pinzado el equipo. Dolor torácico de moderada intensidad. Tos seca que en ocasiones se torna persistente.

\section{Datos positivos al examen físico}

Movimientos respiratorios rápidos y superficiales. Presencia de pleurostomía mínima alta en hemitórax derecho.

Murmullo vesicular disminuido en hemitórax derecho.

Frecuencia cardíaca 115 latidos por minutos.

En la gasometría arterial se constata una ligera hipercapnia, $\mathrm{PaCO}_{2}: 49 \mathrm{mmHg}$.

En el período preoperatorio mediato se indica la realización de rehabilitación respiratoria, inspirometría incentivada y caminatas, con vistas a mejorar la fuerza muscular y mecánica respiratoria. Se inició tra- 
tamiento broncodilatador y dieta baja en carbohidratos.

\section{Técnica anestésica}

Se realiza anestesia peridural con trocar tuhoy 18 G y la colocación de catéter para garantizar la analgesia, tanto intraoperatoria como postoperatoria.

\section{Inducción anestésica}

Se provoca hipnosis con propofol $2 \mathrm{mg} / \mathrm{kg}$. Se administra vecuronio $0,08 \mathrm{mg} / \mathrm{kg}$. De 60 a 90 segundos antes de la intubación se pasa por vía EV lidocaína al $2 \%$ a razón de $1,5 \mathrm{mg} / \mathrm{kg}$.

Se realiza intubación selectiva al pulmón izquierdo con tubo de doble luz tamaño 41 fr comprobándose su correcta colocación.

\section{Mantenimiento anestésico}

Se administró en infusión endovenosa lidocaína a $1,5 \mathrm{mg} / \mathrm{kg} / \mathrm{h}$.

El mantenimiento se realizó con propofol en infusión continua a $6 \mathrm{mg} / \mathrm{kg} / \mathrm{h}$. Se utilizó vecuronio a 0,04 $\mathrm{mg} / \mathrm{kg}$ cada 30 minutos según necesidades. Por el catéter peridural se administró bupivacaína al 0,25\% 10 cc.

Se mantiene la ventilación controlada en modalidad volumen control regulada por presión. Se calcula el volumen tidal a $7 \mathrm{ml}$ por $\mathrm{kg}$ de peso ideal. Se logra mantener presiones medias relativamente bajas incluso durante la ventilación unipulmonar del lado izquierdo, momento en el cual le realiza la bullectomía derecha.

Se mantiene al paciente con la pleurostomía mínima alta izquierda para evitar el neumotórax a tensión que pudiese ocurrir por la rotura de las bullas enfisematosas del pulmón del mismo lado secundarias a los aumentos de las presiones en este pulmón durante la ventilación unipulmonar. De 30 a 40 minutos antes de terminar la operación se le administró al paciente por vía parenteral diclofenaco sódico $75 \mathrm{mg}$.

Se administró bupivacaína al 0,125\% por el catéter peridural antes de terminada la operación.

En el postoperatorio se realiza profilaxis del tromboembolismo pulmonar con fraxiheparina subcutánea. Como cuidados postoperatorios se mantuvo fisioterapia respiratoria, inspirometría incentivada por un mes, ejercicios de baja intensidad y caminatas, con lo que se logró una evolución satisfactoria.

\section{Discusión}

Como estrategia ventilatoria en este paciente utilizamos ventilación controlada en modalidad volumen control regulada por presión. Utilizamos volúmenes bajos ya que la ventilación con volumen corriente (Vc) bajo y la prolongación del tiempo espiratorio evita la hiperinsuflación pulmonar y la rotura de las bulas enfisematosas[5].

Un Vc alto, puede ocasionar sobredistención alveolar e incrementar la resistencia vascular pulmonar, aumentando el corto circuito. Esto ha requerido emplear $\mathrm{Vc}$ menor a $8 \mathrm{~mL} / \mathrm{kg}$ de peso[6].

El principal riesgo que tenía este paciente, era que durante la ventilación a presión positiva y particularmente durante la unipulmonar, hubiese ruptura de una bulla enfisematosa que diese al traste con la vida del mismo. Recordemos que en este caso el paciente presentaba ruptura de bullas bilaterales, y por ende, el pulmón izquierdo (dependiente) había presentado recientemente un neumotórax para lo cual aún tenía colocada una sonda de pleurostomía en posición alta de ese lado.

En cirugía laparoscópica, el neumotórax espontáneo corresponde a una complicación inusual. Eventos como el aumento en las presiones de vía aérea, caída de la $\mathrm{SaO}_{2}$ y cambios en el $\mathrm{ETCO}_{2}$ pueden ser hallazgos tempranos y sutiles durante el desarrollo de esta crisis[7].

La ventilación con presión positiva puede usarse con seguridad en los pacientes con bullas siempre que las presiones en las vías respiratorias se mantengan bajas y que se pueda disponer de inmediato de profesionales expertos y del equipo necesario para insertar un tubo de tórax y lograr el aislamiento pulmonar si fuese necesario[5].

En cuanto a la estrategia de la analgesia postoperatoria, muchas técnicas son mejores que el uso de opiáceos parenterales. Sin embargo, solo las técnicas epidurales disminuyen las complicaciones respiratorias postoracotomía en los pacientes de alto riesgo[8].

Con nuestro paciente nos dimos a la tarea de realizar una técnica anestésica libre de opioides con el propósito de evitar las complicaciones de estos fármacos y garantizar una analgesia más efectiva.

Los efectos adversos de los opioides, son bien conocidos. El uso de las técnicas multimodales ya ha demostrado reducción de estos efectos[9].

El tratamiento multimodal del dolor con opioides vía intravenosa y peridural, anestésicos locales y sulfato de magnesio, permite lograr una adecuada analgesia y la realización de la fisioterapia respiratoria[10]. En el caso de nuestro paciente administramos lidocaína en infusión continua endovenosa, bajas dosis de lidocaína endovenosa están asociadas a una menor incidencia de efectos adversos de los opioides[11].

Oliveira et al.[12], al evaluar el dolor en mujeres 
sometidas a cirugías laparoscópicas en régimen ambulatorio que recibieron la lidocaína en dosis de 1,5 $\mathrm{mg} / \mathrm{kg}$, 20 min antes de la incisión quirúrgica, seguida de una infusión de $2 \mathrm{mg} / \mathrm{kg} / \mathrm{h}$ hasta el final del procedimiento quirúrgico, observan un alivio del dolor en el grupo de la lidocaína.

El dolor torácico crónico postoracotomía se describe en el $20 \%-70 \%$ de los casos[10]. En este paciente no apareció el dolor gracias a terapéutica analgésica multimodal administrada.

En este paciente desde el periodo preoperatorio comenzamos con la realización de fisioterapia respiratoria, ya que los pacientes con EPOC tienen menos complicaciones pulmonares postoperatorias cuando se inicia un programa perioperatorio de fisioterapia respiratoria intensiva antes de la intervención quirúrgica[5].

\section{Conclusiones}

La utilización de estrategias de ventilación controladas en modalidad volumen control regulada por presión. Anestesia combinada y analgesia multimodal, además, de una fisioterapia respiratoria en pacientes que serán operados para realizarles bullectomía, es una buena estrategia anestésica que garantiza una adecuada recuperación del enfermo.

\section{Referencias}

1. GBD 2015 Disease and Injurylncidence and PrevalenceCollaborators. Global,regional, and nationalincidence, prevalence, and yearslivedwithdisabilityfor 310 diseases and injuries, 19902015: a systematicanalysisfortheGlobalBurden of DiseaseStudy 2015. Lancet. 2016; 388:1545602. https://doi.org/10.1016/ s0140-6736(16)31678-6

2. Martínez-Aguilar NE, VargasCamaño ME, Hernández-Pliego RR, Chaia-Semerena GM, PérezChavira MR. Inmunopatología de la enfermedad pulmonar obstructiva crónica. RevAlergMex. 2017;64(3):327-346 https://doi. org/10.29262/ram.v64i3.263

3. Bernard E, Pardo V, Benítez A, Seehaus A. Lesiones pulmonares relacionadas con el tabaquismo. Hallazgos y diagnósticos diferenciales por tomografía computada multidetector. Rev Argent Radiol. 2017;81(4):270-278 https://doi. org/10.1016/j.rard.2017.01.005

4. Mederos Curbelo Orestes Noel, Barrera Ortega Juan Carlos, Guerra Pereda Evelio, Da Costa Fernández José Manuel. Neumotó- rax y bulla enfisematosa gigante rota como manifestación inicial de un carcinoma. Rev cubana Cir [revista en la Internet]. 2010 Dic [citado 2015 Oct 08]; 49(4): 52-57.

5. Chaparro Cruz G, Suguimoto A. Crisis anestésica en cirugía laparoscópica: neumotórax espontáneo bilateral. Diagnóstico y manejo, reporte de caso. Rev colomb anestesiologiol. 2015;4 3(2):163-166 https://doi. org/10.1016/j.rca.2015.01.003

6. Slinger PD, Campos JH. Anestesia para la cirugía torácica. En: Miller RD, editor. Miller Anesthesia. 8va ed. España: Elsevier. 2015; p. 1942-2006.

7. Végh $T$, Juhász $M$, Szatmári $S$, Enyedi A, Sessler DI, Szegedi LL, et al. Effects of differenttidalvolumesforone-lungventilationonoxygenationwith open chestcondition and surgicalmanipulation: a randomisedcross-over trial. Minerva Anestesiol. 2013; 79:24-32.

8. Licker M, Widikker I, Robert J, et al: Operativemortality and respiratorycomplicationsafterlungresectionforcancer: impact of chronicobstructivepulmo- narydisease and time trends, Ann ThoracSurg81:1830, 2006. https://doi.org/10.1016/j.athoracsur.2005.11.048

9. Vargas-Hernández JJ. Anestesia libre de opiodesRev Mexicana de Anestesia. 2014;37(1): 524-7.

10. Ojeda-González J, Paret-Correa N, Ojeda-Delgado L. Consideraciones sobre anestesia en cirugía torácica. Medisur [Internet]. 2013 [citado 7 Nov 2014];11(5): Disponible en: http://medisur. sld.cu/index.php/medisur/article/ view/2568

11. Barros de oliveira $C M$, Kimiko $R$, Slullitel A, Salomao R, Lanchote $V L$, Machado A. Efectos de la lidocaína venosa intraoperatoria sobre el dolor e interlucina- 6 plasmática en pacientes sometidas a histerectomía. RevBras Anestesiol.2015; 65 (2):42-98. https://doi.org/10.1016/j.bjanes.2013.07.018

12. De Oliveira GS, Fitzgerald $P$, Streicher LF. Systemic lidocaine to improve postoperative quality of recovery after ambulatory laparoscopic surgery. AnesthAnalg. 2012; 115:2627. https://doi.org/10.1213/ ANE.0b013e318257a380 